\title{
Relación entre el clima de aprendizaje en la institución educativa y la gestión directiva
}

\author{
Relationship between the learning climate in the educational institution \\ and directive management
}

Erick Joel Hernández Ramos

erickjoel.hernandezramos@gmail.com

Código ORCID: 0000-0002-8097-8716

Universidad Nacional de Piura, Piura, Perú
| Artículo recibido en marzo 2021

| Arbitrado en abril 2021

| Aceptado en abril 2021

| Publicado en mayo 2021

\section{Resumen}

La escuela es una organización compleja, en ella se reúnen diversos factores que exigen de sus directivos excelentes competencias para el manejo de los diferentes aspectos de la institución. Esta investigación se centró en la caracterización de la gestión directiva y el clima de aprendizaje en una institución de educación media en Perú, con el objetivo de establecer la relación entre estas variables y precisar los elementos que contribuyan a optimizar el clima de aprendizaje. Es una investigación confirmatoria, con un diseño de campo. La población estuvo constituida por 42 docentes y 4 directivos. Se usó la encuesta como técnica de recolección de datos y los instrumentos fueron dos cuestionarios. Se planteó como hipótesis que existe relación significativa entre la gestión directiva y el clima de aprendizaje. El estadístico de análisis fue el coeficiente de correlación de Spearman. Se concluye que existe relación significativa entre la gestión directiva y el clima de aprendizaje.

\footnotetext{
Abstract

The school is a complex organization; it brings together various factors that require excellent skills from its managers to manage the different aspects of the institution. This research focused on the characterization of directive management and the learning climate in a secondary education institution in Peru, with the aim of establishing the relationship between these variables and specifying the elements that contribute to optimizing the learning climate. It is a confirmatory investigation, with a field design. The population consisted of 42 teachers and 4 directors. The survey was used as a data collection technique and the instruments were two questionnaires. It was hypothesized that there is a significant relationship between directive management and the learning climate. The statistical analysis was the Spearman correlation coefficient. It is concluded that there is a significant relationship between directive management and the learning climate.
}

\section{Palabras clave:}

Clima de aprendizaje; gestión directiva; gestión escolar; Educación media; educación en Perú

\section{Keywords:}

Learning climate; directive management; school management; secondary education; education in Peru 


\section{INTRODUCCIÓN}

La gestión directiva en la institución escolar está centrada en el logro de la calidad de la educación. Los directivos de una institución educativa están abocados contantemente, en su gestión, al alcance de metas para el logro de la calidad de la educación. Los procesos educativos deben permitir obtener resultados positivos para que los estudiantes adquieran las competencias necesarias y consigan desenvolverse en cualquier ámbito que les corresponda actuar. Para ello la gestión educativa debe atender los diferentes factores que contribuyen para alcanzar un óptimo clima de aprendizaje.

El clima de aprendizaje es objeto de estudio de numerosas investigaciones entre los cuales se encuentra el análisis del clima escolar realizado por la UNESCO en el 2013 reveló que tal como en los ecosistemas biológicos, el clima escolar suele ser reflejo de las interacciones que ocurren dentro de la escuela y de las condiciones en las instituciones educativas. Se acepta que para que exista un clima escolar positivo es necesaria la cooperación entre directivos, administrativos, personal de apoyo y profesores, en la que participan las familias y los estudiantes, todos ejecutando sus funciones para conseguir la calidad educativa. Las relaciones que se desarrollen entre todos los integrantes de la comunidad educativa deben estar centradas en el respeto, la confianza y la participación democrática. De esta forma se puede inferir que la definición de clima de aprendizaje debe tomar en cuenta la percepción de todos los actores involucrados, y sus respectivas interacciones. Autores como Adelman y Taylor (2004); Herrera y Rico (2014) Espinoza y Rodríguez (2017) coinciden en señalar que el clima de aprendizaje es el factor clave que define y caracteriza una escuela eficaz y se caracteriza por la buenas relaciones que a su vez causan efectos positivos como el compromiso y la valoración del trabajo en equipo desde la percepción que tienen los integrantes de la comunidad educativa. Murillo (2011) agrega que el ambiente educativo adecuado contribuye a desarrollar la autoestima y un sentido de pertenencia e identidad con la clase y la escuela.

Las dimensiones para medir el clima de aprendizaje según Molina y Pérez (2006), Blanco (2014) y García y Ferreira (2005) son: el contexto interpersonal, ambientes de aprendizajes acogedores y ambientes saludables de aprendizaje.

Se hace necesario entonces que las personas responsables de la gestión en la institución educativa, tengan las competencias para fomentar las relaciones entre los diferentes niveles que conforman el triángulo educativo: docentes, estudiantes y padres de familia para que el clima escolar se presente de una manera positiva para todos los componentes de la institución. Desde la perspectiva del liderazgo pedagógico, según el Ministerio de Educación del Perú (2015) es primordial que el equipo directivo cuente con la capacidad técnica, el manejo emocional, situacional y organizacional como puntos importantes en la gestión del director.

La gestión directiva escolar se fundamenta en los procesos administrativos planificación, organización, dirección y control. Estos procesos permiten organizar la formulación del Proyecto Educativo Institucional y plantear el Plan Operativo Anual, con fundamento en la correspondiente autoevaluación institucional, y a partir de ello, lograr la consolidación de un plan de mejoramiento, que incluya estrategias, proyectos, planes de trabajo (Ruiz y Gallo, 2011).

La Escuela Agroecológica de Pirqué de Chile (sf) agrega que las áreas de la gestión escolar son: gestión académica, gestión directiva, gestión administrativa, y gestión de la comunidad (p.2). Entre las principales funciones de la gestión directiva se tiene: (a) Gestionar el currículum y los elementos curriculares, (b) Liderar las relaciones humanas, (c) Gestionar los procesos pedagógicos, (d) Gestionar la convivencia democrática e intercultural, (e) Gestionar el vínculo entre la escuela, la familia y la comunidad. 
En la Institución Educativa de educación media "Federico Helguero" Seminario de Los Algarrobos en Piura existe preocupación por tener el mejor clima de aprendizaje, el interés de esta investigación es la caracterización de la gestión directiva y el clima de aprendizaje en esta institución de educación media en Perú, con el objetivo de establecer la relación entre estas variables y precisar los elementos que contribuyan a optimizar el clima de aprendizaje.

La hipotesis de investigación y la hipótesis nula son:

Hi: Existe relación significativa entre la gestión directiva y el clima de aprendizaje en la Institución Educativa "Federico Helguero" Seminario de los Algarrobos

H0: No existe relación significativa entre la gestión directiva y el clima de aprendizaje en la Institución Educativa "Federico Helguero" Seminario de los Algarrobos

\section{MÉTODO}

Es una investigación confirmatoria, con un diseño de campo, la población estuvo constituida por 42 docentes y 4 directivos de la Institución Educativa "Federico Helguero" Seminario de los Algarrobos en Piura, Perú. Se usó la encuesta como técnica de recolección de datos y los instrumentos fueron dos cuestionarios, uno para describir la gestión directiva con 41 ítems y el otro para la caracterización del clima de aprendizaje con 23 ítems; ambos con tipo de respuesta tipo Likert. Estos instrumentos fueron validados por juicios de expertos y la confiabilidad se calculó con el Alfa de Cronbach. Siendo los valores de confiabilidad 0,790, indicando una aceptable validez en el primer instrumento, y 0,847 indicando una validez elevada.

\section{RESULTADOS}

Descripción de la gestión directiva

\section{Gestión de recursos pedagógicos}

Cuadro 1. Gestión de recursos pedagógicos.

\begin{tabular}{lcc}
\hline \multicolumn{1}{c}{ Ítems } & Media & Desviación estándar \\
\hline D1: Gestión de recursos pedagógicos & &, 816 \\
1. Mantienen una adecuada provisión de materiales visuales & 2,85 &, 750 \\
2. Mantienen una adecuada provisión de materiales auditivos & 2,72 &, 726 \\
3. Mantienen una adecuada provisión de materiales audiovisuales & 2,70 &, 750 \\
4. Mantienen una adecuada organización de materiales visuales & 2,70 &, 720 \\
5. Mantienen una adecuada organización de materiales auditivos & 2,72 &, 767 \\
6. Mantienen una adecuada organización de materiales audiovisuales & 2,72 &, 814 \\
7. Mantienen un adecuado uso de materiales visuales & 2,89 &, 687 \\
8. Mantienen un adecuado uso de materiales auditivos & 2,78 & 2,87 \\
\hline
\end{tabular}




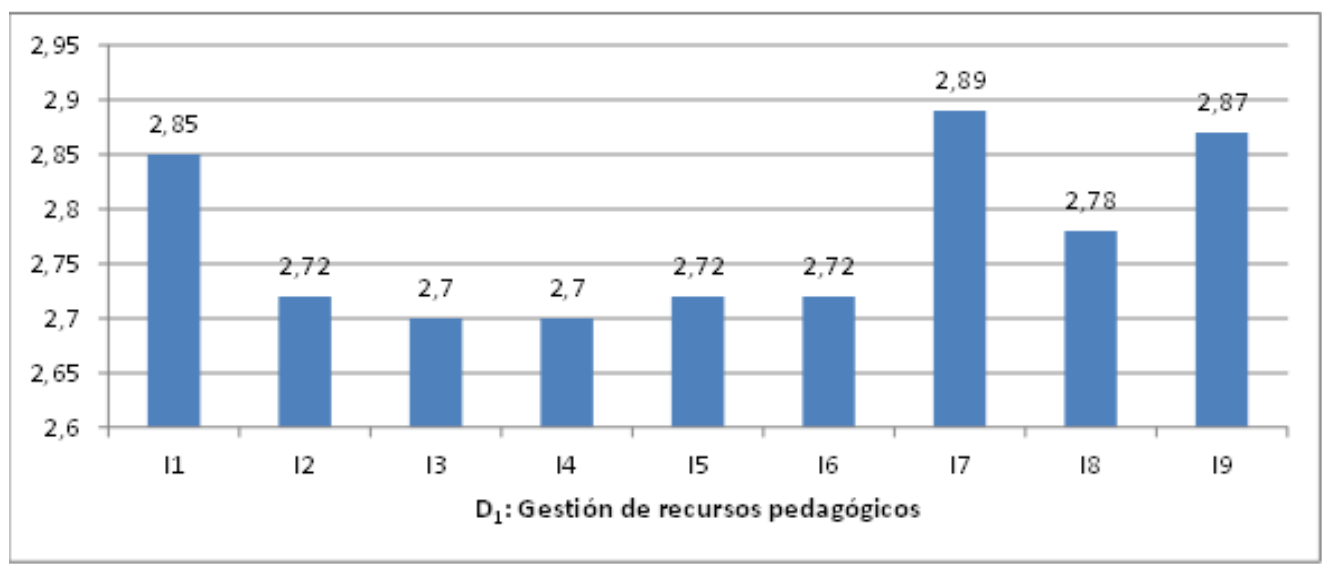

Gráfico 1. Gestión de recursos pedagógicos.

De acuerdo a la tabla anterior, los docentes y directivos encuestados perciben que la gestión directiva escolar cumple con la gestión de los recursos pedagógicos. Así se registra en los puntajes promedio próximos a 3 puntos. En resumen, la dirección escolar mantiene una adecuada provisión, organización y uso de materiales visuales, auditivos y audiovisuales.

\section{Atención a las necesidades de los estudiantes}

Cuadro 2. Atención a las necesidades de los estudiantes.

\begin{tabular}{lcc}
\hline \multicolumn{1}{c}{ Ítems } & Media & Desviación estándar \\
\hline D2: Atiende las necesidades de los estudiantes & \\
$\begin{array}{l}\text { 10. Mantienen prácticas para apoyar el desarrollo progresivo de los } \\
\text { estudiantes atendiendo a las dificultades y avances en su aprendizaje }\end{array}$ & 3,22 &, 664 \\
$\begin{array}{l}\text { 1. Mantienen prácticas para facilitar el desarrollo cognitivo de los } \\
\text { estudiantes, considerando sus características y necesidades }\end{array}$ & 3,04 &, 665 \\
$\begin{array}{l}\text { 12. Mantienen prácticas que aseguran la el desarrollo socioemocional, } \\
\text { considerando sus características y necesidades }\end{array}$ & 3,07 &, 712 \\
\hline
\end{tabular}

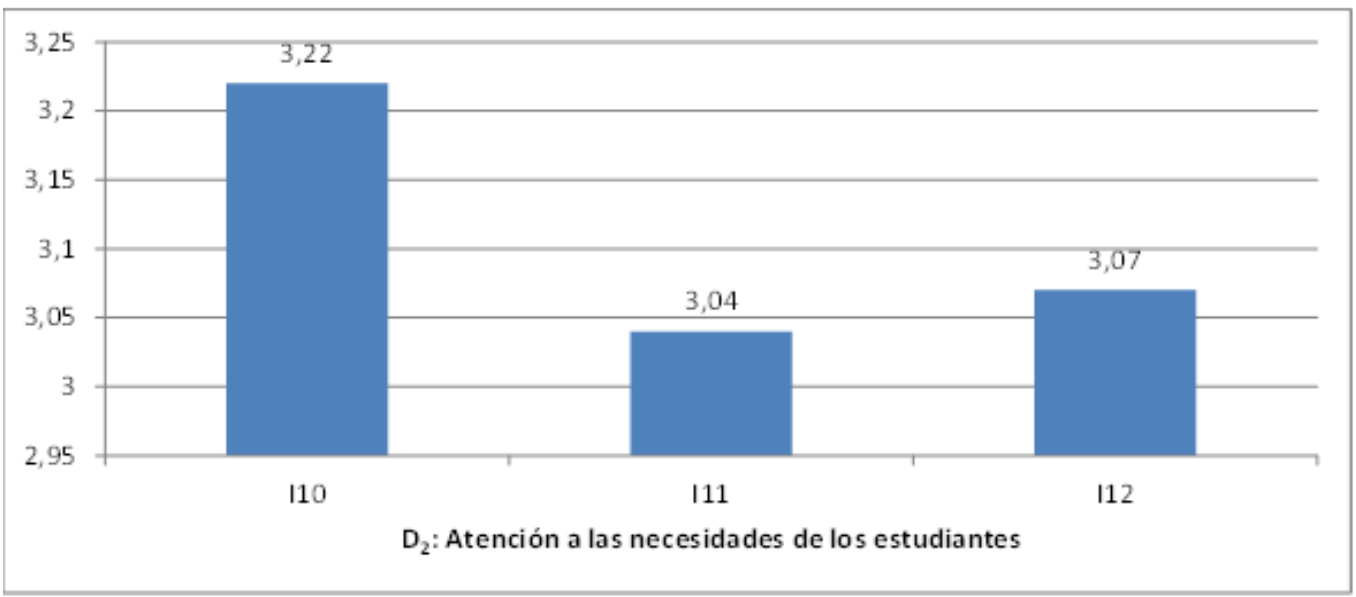

Gráfico 2. Atención a las necesidades de estudiantes. 
Según la tabla anterior, los docentes y directivos encuestados observan que la gestión directiva escolar cumple con atender las necesidades pedagógicas de los estudiantes. Así se extrae en los puntajes promedio próximos a 3 puntos. En efecto la dirección escolar ejecuta prácticas para apoyar el desarrollo progresivo de los estudiantes atendiendo a sus dificultades y avances en su aprendizaje, facilita el desarrollo cognitivo de los estudiantes tomando en cuenta sus características y necesidades, igual consideración para el desarrollo socioemocional de los escolares.

\section{Gestión de estrategias didácticas}

Cuadro 3. Gestión de estrategias didácticas.

\begin{tabular}{lcc}
\hline \multicolumn{1}{c}{ Ítems } & Media & Desviación estándar \\
\hline D3: Gestión de estrategias didácticas & & \\
$\begin{array}{l}\text { 13. Mantienen prácticas para asegurar que las estrategias de enseñanza } \\
\text { diseñadas por los docentes son pertinentes y coherentes a las necesidades } \\
\text { de los estudiantes. }\end{array}$ & 3,07 &, 772 \\
$\begin{array}{l}\text { 14. Mantienen prácticas para asegurar que los recursos educativos e } \\
\text { informáticos se utilizan en coherencia con las estrategias de enseñanza } \\
\text { diseñadas por los docentes. }\end{array}$ & 3,00 &, 632 \\
$\begin{array}{l}\text { 15. Mantienen prácticas que aseguran la coherencia entre los procedi- } \\
\text { mientos de evaluación de los aprendizajes y las estrategias de enseñanza } \\
\text { diseñadas por los docentes. }\end{array}$ & 3,00 &, 699 \\
\hline
\end{tabular}

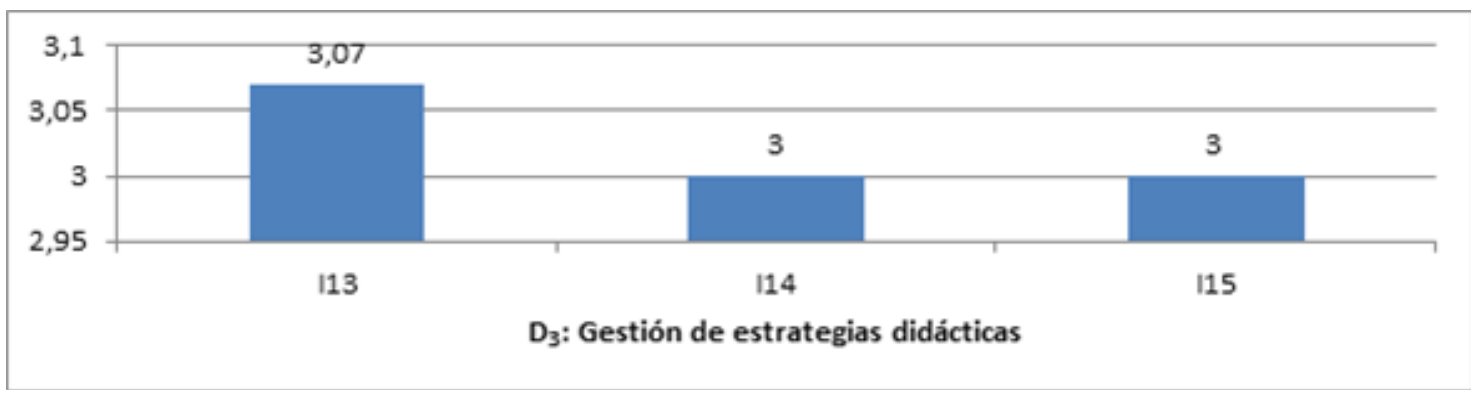

Gráfico 3. Gestión de estrategias didácticas.

De acuerdo a la tabla anterior, los docentes y directivos encuestados observan que la gestión directiva escolar cumple con gestionar las estrategias didácticas. Así se obtiene de los puntajes promedio de 3 puntos o los resultados próximos. En consecuencia, la dirección escolar asegura que las estrategias de enseñanza diseñadas por los docentes sean pertinentes y coherentes con las necesidades de los escolares, se preocupa por el buen uso de los recursos informático en las sesiones de aprendizaje, y procura que la evaluación de los aprendizajes y las estrategias de enseñanza sean coherentes con las áreas curriculares. 


\section{Gestión de la organización escolar}

Cuadro 4. Gestión de la organización escolar.

\begin{tabular}{lcc}
\hline \multicolumn{1}{c}{ Ítems } & Media & Desviación estándar \\
\hline D4: Gestión de la organización escolar & &, 778 \\
$\begin{array}{l}\text { 16. Mantienen prácticas que aseguran la formación continua de los } \\
\text { docentes y para docentes de acuerdo a sus funciones y metas establecidas }\end{array}$ & 2,80 &, 706 \\
$\begin{array}{l}\text { 17. Mantienen prácticas que aseguran la evaluación de desempeño } \\
\text { de los docentes y para docentes de acuerdo a sus funciones y Metas } \\
\text { establecidas }\end{array}$ & 2,89 \\
$\begin{array}{l}\text { 18. Trabajan coordinadamente con la AAPAFA } \\
\text { 19. Mantienen prácticas que aseguran la planificación institucional y } \\
\text { curricular }\end{array}$ & 3,02 \\
$\begin{array}{l}\text { 20. Mantienen prácticas que aseguran el seguimiento de las actividades } \\
\text { educativas }\end{array}$ & 2,65 \\
$\begin{array}{l}\text { 21. Mantienen prácticas que aseguran las relaciones con la comunidad } \\
\text { educativa. }\end{array}$
\end{tabular}

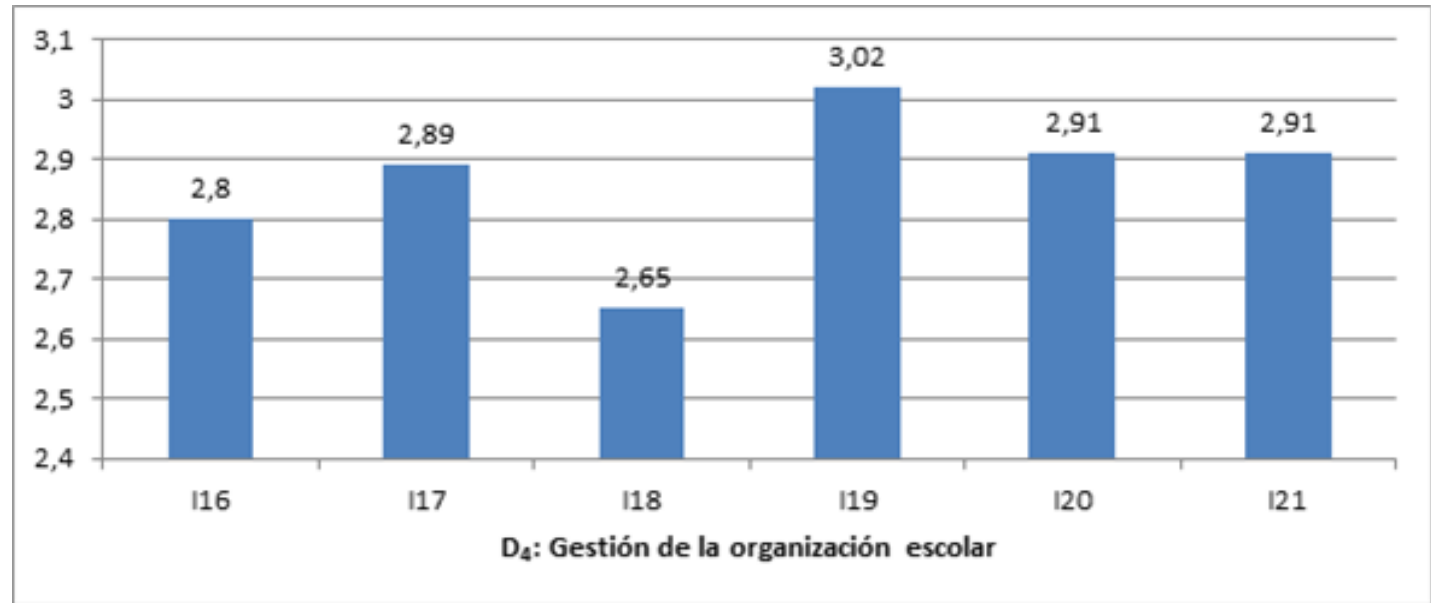

Gráfico 4. Gestión de la organización escolar.

Según la tabla anterior, los docentes y directivos encuestados perciben que la gestión directiva escolar cumple con gestionar la organización escolar. Así se explora de los puntajes promedio de 3 puntos o cercanos a este. En efecto la dirección escolar incentiva la formación continua de sus docentes, evalúa el desempeño docente, trabaja coordinadamente con la asociación de padres de familia, asegura que la planificación institucional y curricular se cumpla, asegura el seguimiento de las actividades educativas y mantiene comunicación con la comunidad educativa. 


\section{Gestión del trabajo administrativo}

Cuadro 5. Gestión del trabajo administrativo.

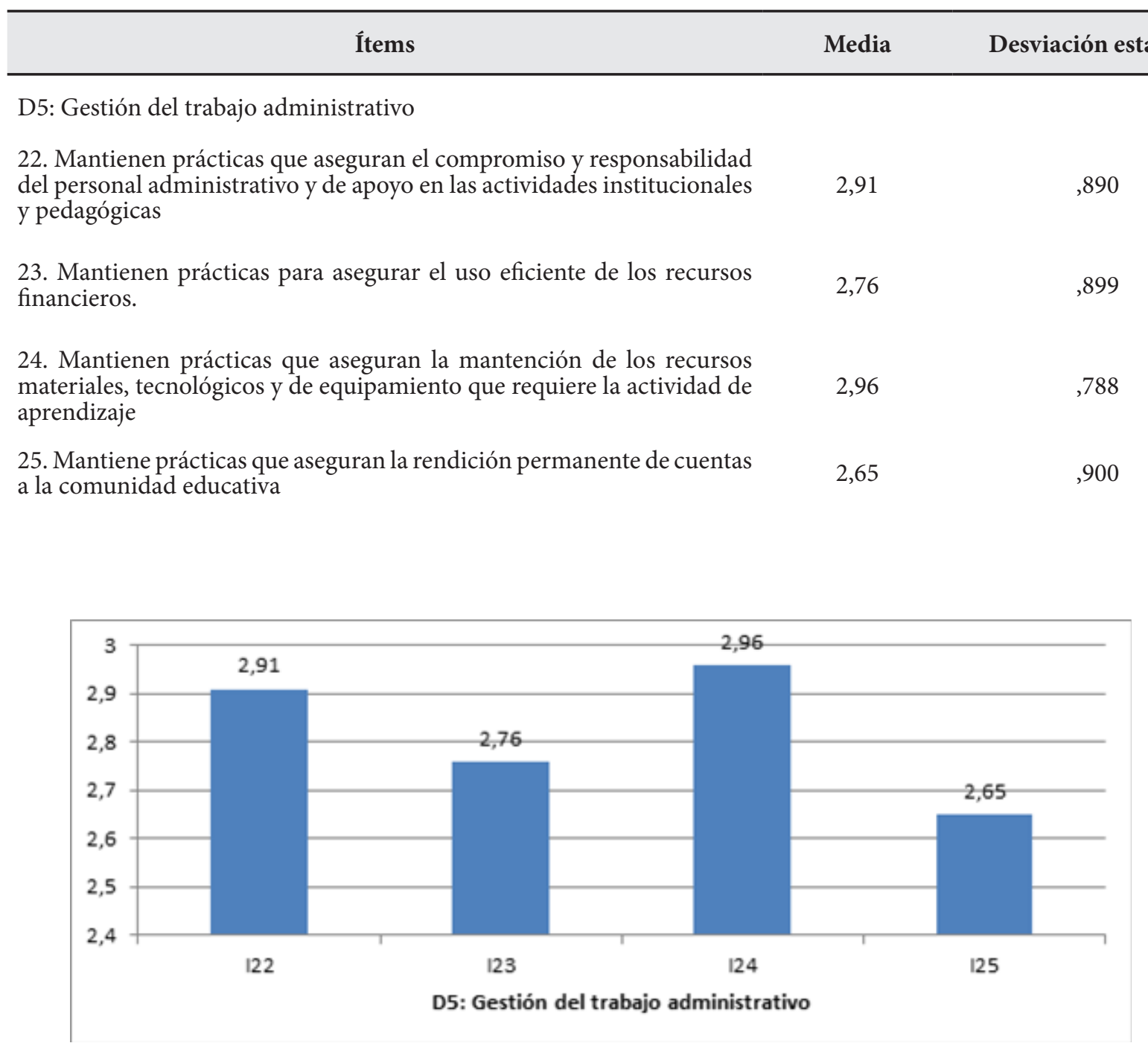

\section{Gráfico 5. Gestión del trabajo administrativo}

Según la tabla anterior, los docentes y de acuerdo a la tabla anterior, los docentes y directivos encuestados observan que la gestión directiva escolar cumple con la gestión administrativa. Así se obtiene de los puntajes promedio cercanos a 3 puntos. En consecuencia, la dirección escolar ejecuta prácticas que aseguran el compromiso y responsabilidad del personal administrativo y de apoyo institucional y pedagógico, asegura la eficiencia de los recursos financieros, da mantenimiento a los recursos materiales, tecnológicos y de equipamiento y mantiene informado a la comunidad educativa de las actividades que realiza la institución. 


\section{Gestión de la organización escolar y la comunidad}

Cuadro 6. Gestión de la organización escolar y comunidad.

Ítems Media Desviación estándar

D6: Gestión de la organización escolar y la comunidad

26. Mantienen prácticas para establecer redes de colaboración con organismos de la comunidad local, académicas, servicios públicos y/o empresariales, entre otros, para apoyar el logro de los Objetivos y Metas Institucionales

27. Mantienen prácticas para asegurar que el involucramiento de padres y/o familias está en función de la implementación del PEI y del apoyo a los aprendizajes de sus hijos.

28. Mantienen prácticas que aseguran la información y canales expeditos de comunicación para mantener informados a todos los actores de la comunidad educativa y recibir observaciones y sugerencias.

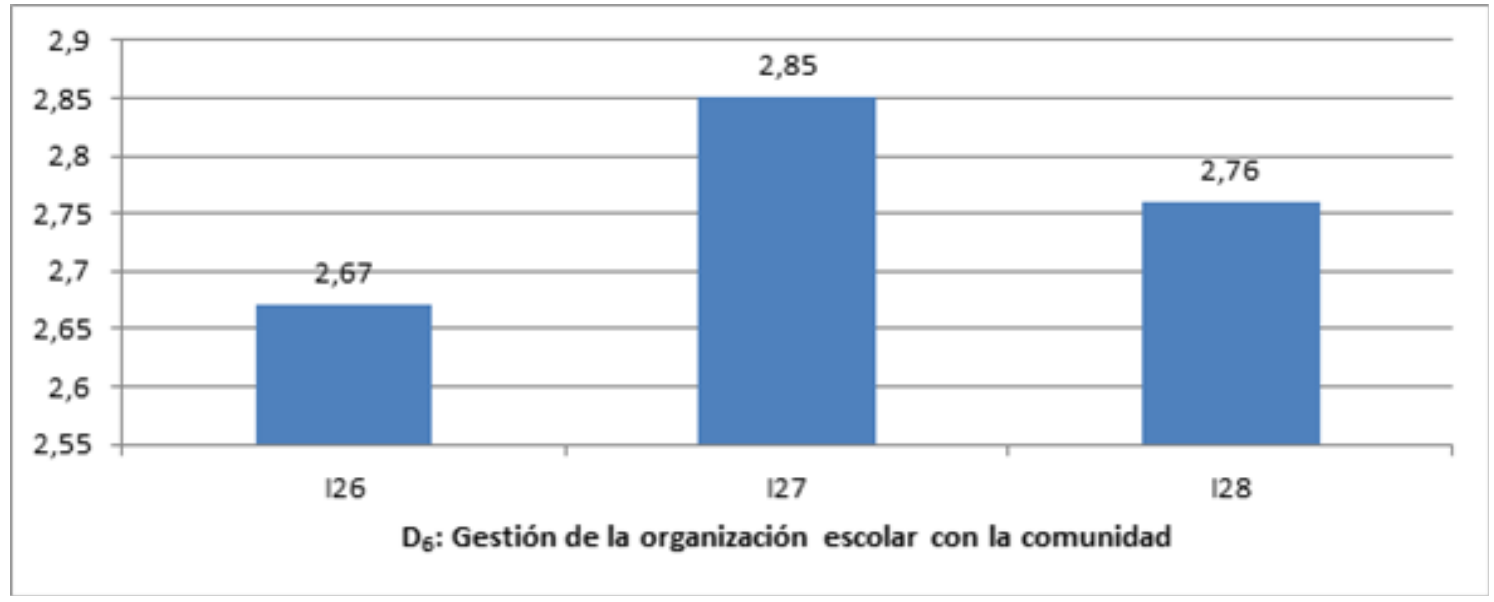

Gráfico 6. Gestión de la organización escolar con la comunidad.

Según la tabla anterior, los docentes encuestados observan que la gestión directiva escolar cumple con gestionar la organización escolar y la comunidad. Así se registra de los puntajes promedio cercanos a 3 puntos. En consecuencia, la dirección escolar establece redes de colaboración con la municipalidad local, instituciones académicas, empresas para incorporarlos en el trabajo escolar, involucra a los padres de familia en el PEI y el aprendizaje de sus hijos, y mantiene comunicación con la comunidad educativa. 


\section{Liderazgo en la organización escolar}

Cuadro 7. Liderazgo en la organización escolar.

\begin{tabular}{|c|c|c|}
\hline Ítems & Media & Desviación estándar \\
\hline \multicolumn{3}{|l|}{ D7: Liderazgo en la organización escolar } \\
\hline $\begin{array}{l}\text { 29. Mantienen prácticas de diálogo permanente con la comunidad } \\
\text { educativa }\end{array}$ & 2,93 & ,772 \\
\hline $\begin{array}{l}\text { 30. Mantienen prácticas de comunicación permanente con la comunidad } \\
\text { educativa }\end{array}$ & 3,00 &, 760 \\
\hline $\begin{array}{l}\text { 31. Mantienen prácticas de tolerancia permanente con la comunidad } \\
\text { educativa }\end{array}$ & 2,96 & ,788 \\
\hline $\begin{array}{l}\text { 32. Mantienen prácticas de aplicación normativa e con toda la } \\
\text { comunidad educativa }\end{array}$ & 3,04 & ,665 \\
\hline $\begin{array}{l}\text { 33. Tienen una visión clara de lo que puede llegar a ser la escuela y ésta } \\
\text { es compartida por el equipo docente. }\end{array}$ & 2,96 & ,698 \\
\hline $\begin{array}{l}\text { 34. Conocen ampliamente las necesidades de la institución, relacionadas } \\
\text { con el aprendizaje de los estudiantes y hacen las gestiones necesarias } \\
\text { para subsanarlas. }\end{array}$ & 3,04 & ,759 \\
\hline $\begin{array}{l}\text { 35. Coordinan la implementación del currículum y vigilan que los } \\
\text { contenidos se desarrollen totalmente. }\end{array}$ & 3,11 & ,767 \\
\hline $\begin{array}{l}\text { 36. Implementan acciones para el acompañamiento pedagógico, lo } \\
\text { que implica observar el trabajo de los docentes en el aula para ofrecer } \\
\text { retroalimentación. }\end{array}$ & 3,11 & ,795 \\
\hline $\begin{array}{l}\text { 37. Promueven el monitoreo y evaluación del aprendizaje de los } \\
\text { estudiantes. }\end{array}$ & 3,26 & ,773 \\
\hline $\begin{array}{l}\text { 38. Promueven un buen ambiente de trabajo donde priva la seguridad } \\
\text { y el orden }\end{array}$ & 3,20 & 833 \\
\hline $\begin{array}{l}\text { 39. Promueven un clima de aprendizaje académico estableciendo } \\
\text { expectativas y estándares positivos. }\end{array}$ & 3,04 & 842 \\
\hline $\begin{array}{l}\text { 40. Promueven la participación de los padres en torno a la educación de } \\
\text { los estudiantes. }\end{array}$ & 3,00 & 869 \\
\hline $\begin{array}{l}\text { 41. Dedican tiempo y energía para implementar programas de } \\
\text { mejoramiento. }\end{array}$ & 2,89 & 849 \\
\hline
\end{tabular}

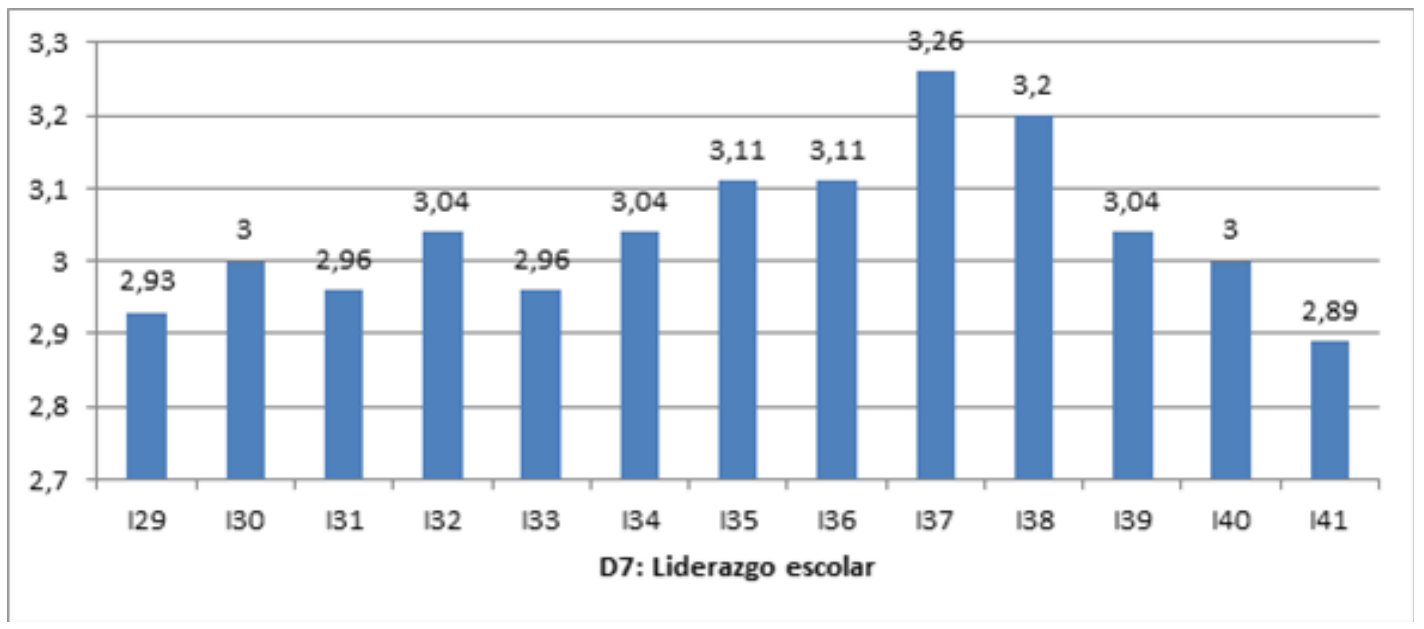

Gráfico 7. Liderazgo en la organización escolar. 
De acuerdo a la tabla anterior, los docentes y directivos encuestados observan que la gestión directiva escolar cumple con el liderazgo institucional. Así se registra de los puntajes promedio cercanos a 3 puntos. En efecto la dirección escolar ejecuta diálogos con la comunidad educativa, mantiene cales de comunicación, practica la tolerancia, cumple con las normas, tiene visión institucional, cumple resolviendo necesidades institucionales, vigila el cumplimiento de programación curricular, ejecuta el monitoreo y acompañamiento pedagógico, promueve un ambiente de trabajo adecuado con clima positivo para el aprendizaje, involucra a los padres de familia en el aprendizaje escolar y ejecuta programas de mejoramiento académico.

\section{Gestión directiva}

Cuadro 8. Global de gestión directiva.

\begin{tabular}{|c|c|c|c|c|c|c|c|c|}
\hline \multirow{2}{*}{ Variable/Dimensiones } & \multicolumn{2}{|c|}{ Ineficiente } & \multicolumn{2}{|c|}{ Poco eficiente } & \multicolumn{2}{|c|}{ Eficiente } & \multicolumn{2}{|c|}{ Total } \\
\hline & $\mathbf{n}^{\circ}$ & $\%$ & $\mathrm{n}^{\mathbf{o}}$ & $\%$ & $\mathbf{n}^{\mathbf{o}}$ & $\%$ & $\mathbf{n}^{\mathbf{o}}$ & $\%$ \\
\hline V1: Gestión directiva & 3 & 6,5 & 22 & 47,8 & 21 & 45,7 & 46 & 100,0 \\
\hline D1: Gestión de recursos pedagógicos & 8 & 17,4 & 27 & 58,7 & 11 & 23,9 & 46 & 100,0 \\
\hline D2: Atiende a las necesidades de los estudiantes & 3 & 6,5 & 26 & 56,7 & 17 & 37,0 & 46 & 100,0 \\
\hline D3: Gestión estrategias didáctica & 0 & 0 & 5 & 10,9 & 41 & 89,1 & 46 & 100,0 \\
\hline D4: Gestión a la organización escolar & 5 & 10,9 & 27 & 58,7 & 14 & 30,4 & 46 & 100,0 \\
\hline D5: Gestión del trabajo administrativo & 8 & 17,4 & 23 & 50,0 & 15 & 32,6 & 46 & 100,0 \\
\hline $\begin{array}{l}\text { D6: Gestión entre la organización escolar y la } \\
\text { comunidad }\end{array}$ & 11 & 23,9 & 25 & 54,3 & 10 & 21,7 & 46 & 100,0 \\
\hline D7: Liderazgo escolar & 3 & 6,5 & 22 & 47,8 & 21 & 45,7 & 46 & 100,0 \\
\hline
\end{tabular}

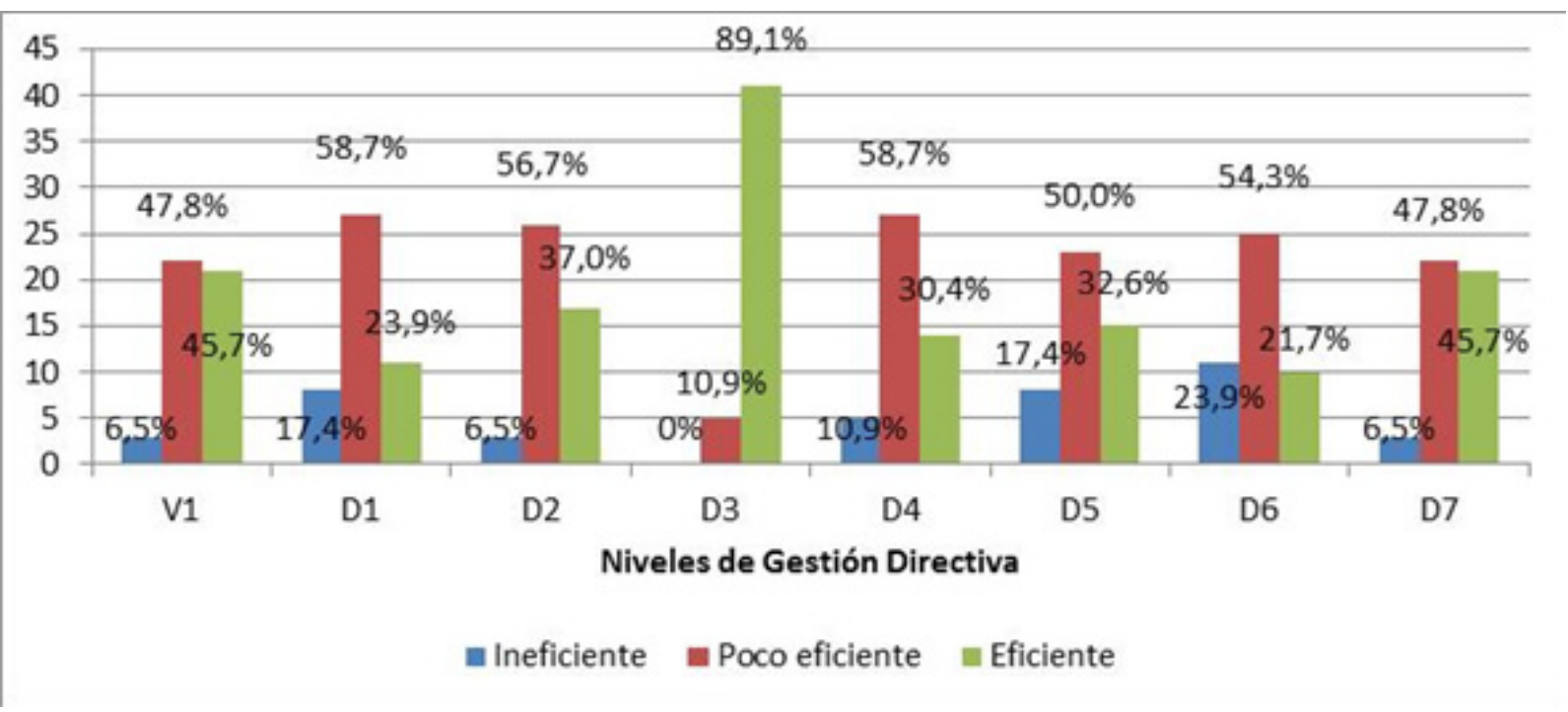

Gráfico 8. Global de gestión directiva. 
Para analizar la gestión directiva, se ha tenido en cuenta aspectos relacionados a la gestión de recursos pedagógicos, atención a las necesidades estudiantiles, gestión de las estrategias didácticas, gestión de la organización escolar, gestión del trabajo administrativo, gestión entre la organización escolar y la comunidad, y el liderazgo escolar; los promedios cercanos o muy próximos a 3 puntos a esta cifra, indica que la percepción de los docentes y directivos es que la gestión directiva cumple con todos ellos.

Al evaluar el nivel de gestión directiva escolar, el estudio indica que para el $47,8 \%$ y $45,7 \%$ de docentes y directivos, la gestión directiva es poco eficiente y eficiente, lo que explica por las cifras altas de estos niveles en la gestión de recursos pedagógicos, atención a las necesidades estudiantiles, gestión de las estrategias didácticas, gestión de la organización escolar, gestión del trabajo administrativo, gestión entre la organización escolar y la comunidad, y el liderazgo escolar. No obstante, en 6 de las 7 dimensiones estudiadas, los docentes aprecian niveles deficientes $\mathrm{D}_{1}=17,4 \%$; $\mathrm{D}_{2}=6,5 \%$; $\mathrm{D}_{4}=$ $10,9 \%$; $\mathrm{D}_{5}=17,4 \%$; $\mathrm{D} 6=23,9 \%$ y $\mathrm{D}_{7}=6,5 \%$.

A continuación se presentan los resultados del clima de aprendizaje

Cuadro 9. Global niveles de clima de aprendizaje.

\begin{tabular}{lccccccccc}
\hline \multirow{2}{*}{\multicolumn{1}{c}{ Variable/Dimensiones }} & \multicolumn{3}{c}{ Bajo } & \multicolumn{2}{c}{ Medio } & \multicolumn{2}{c}{ Alto } & \multicolumn{2}{c}{ Total } \\
\cline { 2 - 10 } & $\mathbf{n}^{\mathbf{0}}$ & $\%$ & $\mathbf{n}^{\mathbf{0}}$ & $\%$ & $\mathbf{n}^{\mathbf{0}}$ & $\%$ & $\mathbf{n}^{\mathbf{o}}$ & $\%$ \\
\hline V2: Clima de aprendizaje & 0 &, 0 & 10 & 21,7 & 36 & 78,3 & 46 & 100,0 \\
D1: Relaciones interpersonales & 0 &, 0 & 15 & 32,6 & 31 & 67,4 & 46 & 100,0 \\
D2: Promueve ambientes de aprendizaje acogedores & 0 &, 0 & 16 & 34,8 & 30 & 65,2 & 46 & 100,0 \\
D3: Ambientes saludables de aprendizaje & 0 &, 0 & 15 & 32,6 & 31 & 67,4 & 46 & 100,0 \\
\hline
\end{tabular}

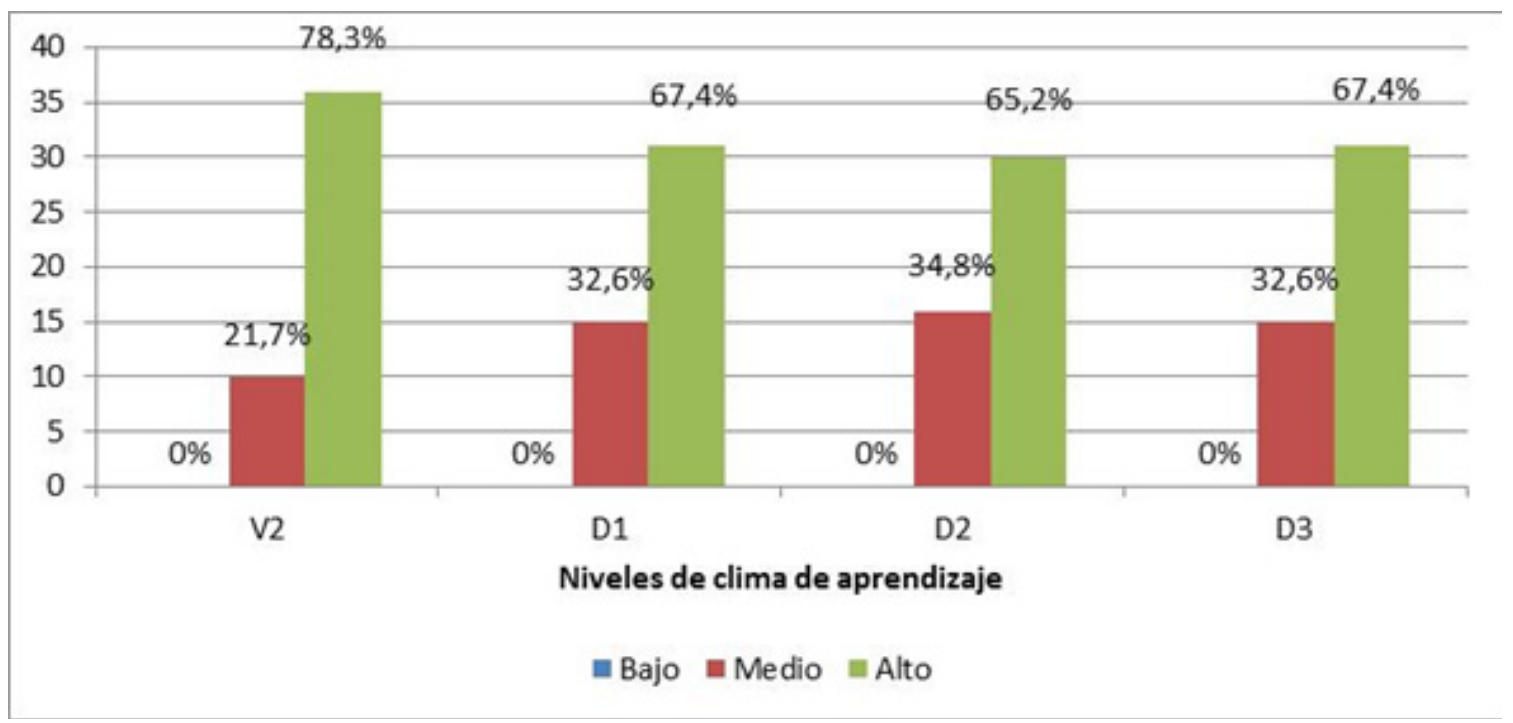

Gráfico 9. Niveles de clima de aprendizaje. 
Al evaluar el nivel de cima de aprendizaje, el estudio indica que para el $78,3 \%$ de docentes y directivos, el clima de aprendizaje es alto, lo que explica por las cifras altas en las relaciones interpersonales, ambiente de aprendizaje acogedor y ambiente saludable de aprendizaje, cada uno con $67,4 \%, 65,2 \%$ y $67,4 \%$, respectivamente. No obstante, un $21,7 \%$ reconoce la variable en un término medio, donde también se justifica por las cifras en un nivel medio $\mathrm{D} 1=32,6 \%$; $2=34,8 \%$ y D3=32,6\%.

\section{Gestión directiva y el clima de aprendizaje.}

Cuadro 10. Relación entre la gestión directiva y las relaciones interpersonales.

\begin{tabular}{|c|c|c|c|c|c|c|c|c|}
\hline \multirow{3}{*}{ D1: Gestión Directiva } & \multicolumn{8}{|c|}{ V2: Clima de aprendizaje } \\
\hline & \multicolumn{2}{|c|}{ Bajo } & \multicolumn{2}{|c|}{ Medio } & \multicolumn{2}{|c|}{ Alto } & \multicolumn{2}{|c|}{ Total } \\
\hline & no $^{\circ}$ & $\%$ & no $^{\circ}$ & $\%$ & $\mathbf{n}^{\mathbf{o}}$ & $\%$ & $\mathbf{n}^{\circ}$ & $\%$ \\
\hline Deficiente & 0 & ,0 & 1 & 33,3 & 2 & 66,7 & 3 & 100,0 \\
\hline Poco eficiente & 0 & , 0 & 6 & 27,3 & 16 & 72,7 & 22 & 100,0 \\
\hline Eficiente & 0 & ,0 & 3 & 14,3 & 18 & 85,7 & 21 & 100,0 \\
\hline Total & 0 & 0 & 10 & 21,7 & 36 & 78,1 & 46 & 100,0 \\
\hline
\end{tabular}

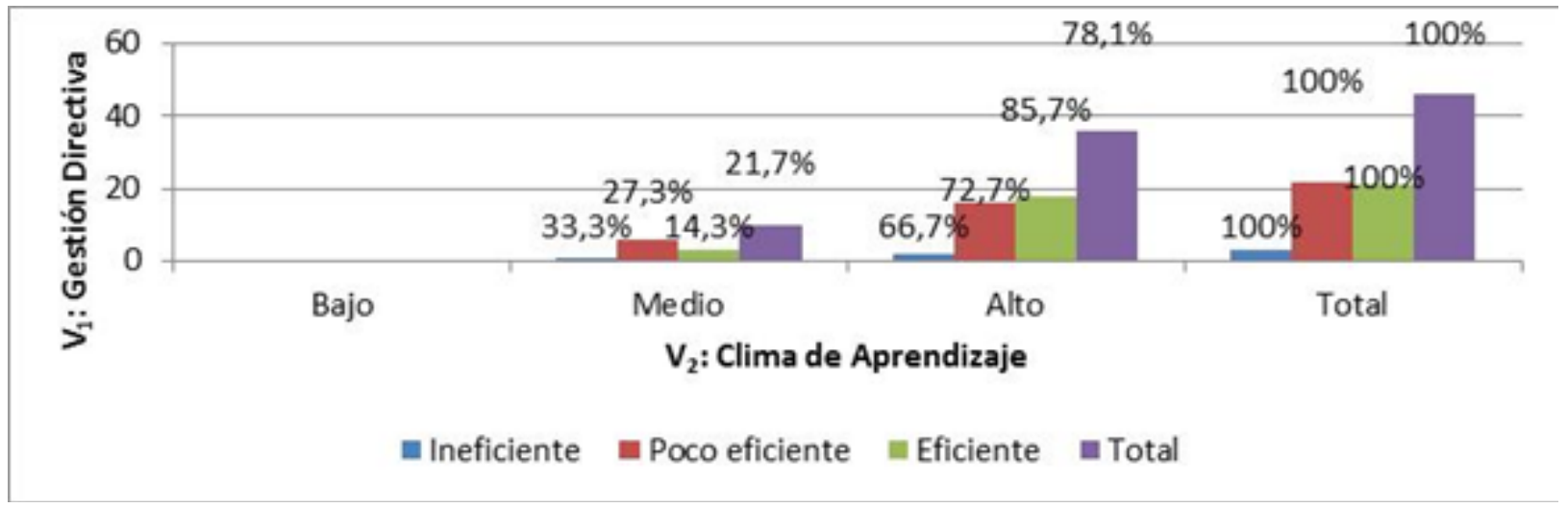

Gráfico 10. Relación entre la gestión directiva y las relaciones interpersonales.

Los resultados de la investigación dejan en evidencia que $1(33,3 \%)$ docentes y directivos que perciben un nivel ineficiente de gestión directiva, califica el clima de aprendizaje en un nivel medio y $2(66,7 \%)$; en tanto $6(27,3 \%)$ docentes y directivos que perciben un nivel poco eficiente de gestión directiva, califican el clima de aprendizaje en un nivel medio y 16 $(72,7 \%)$ en un nivel alto. Por otro lado, 3 (14,3\%) docentes y directivos que perciben un nivel eficiente de gestión directiva, califican el clima de aprendizaje en un nivel medio y 18 (85,7\%) en un nivel alto. 


\section{Contrastación de hipótesis}

\section{Contrastación de la hipótesis general}

Hi: Existe relación significativa entre la gestión directiva y el clima de aprendizaje en la Institución Educativa "Federico Helguero" Seminario de los Algarrobos.
H0: No existe relación significativa entre la gestión directiva y el clima de aprendizaje en la Institución Educativa "Federico Helguero" Seminario de los Algarrobos.

Cuadro 11. Correlación entre la gestión directiva y el clima de aprendizaje.

\begin{tabular}{ccc} 
& Spearman & $\mathrm{V}_{2}$ : Clima de aprendizaje \\
\hline & $\mathrm{r}$ &, $359^{\star}$ \\
$\mathrm{V}_{1}$ : Gestión Directiva & Sig. &, 014 \\
& $\mathrm{n}^{\circ}$ & 46 \\
\hline
\end{tabular}

* La correlación es significativa en el nivel 0,05 (2 colas)

El análisis de correlación indica que la gestión directiva se relaciona de manera significativa (Sig. $<0.05)$ Con el clima de aprendizaje; este resultado da evidencias suficientes para validar la hipótesis de investigación. Este resultado permite establecer que en la medida que mejora la gestión directiva escolar mejorará el clima de aprendizaje.

\section{Contrastación de las hipótesis específicas}

\section{Gestión directiva y relaciones interpersonales}

Hi1: Existe relación entre la gestión directiva y las relaciones interpersonales.

H01: No existe relación entre la gestión directiva y las relaciones interpersonales.

Cuadro 12. Correlación entre gestión directiva y las relaciones interpersonales

\begin{tabular}{ccc} 
& Spearman & $\mathrm{D}_{1}$ : Relaciones interpersonales \\
\hline & $\mathrm{r}$ &, $406^{* *}$ \\
$\mathrm{~V}_{1}$ : Gestión Directiva & Sig. &, 004 \\
& $\mathrm{n}^{\circ}$ & 46 \\
\hline
\end{tabular}

** La correlación es significativa en el nivel 0,01 (2 colas). 
Elanálisis de correlación indica que la gestión directiva se relaciona de manera significativa (Sig. <0.05) Con las relaciones interpersonales; este resultado da evidencias suficientes para validar la hipótesis de investigación. Este resultado permite establecer que en la medida que mejora la gestión directiva escolar mejorará las relaciones interpersonales.

\section{Gestión directiva y ambientes de aprendizaje acogedores}

Hi2: Existe relación entre la gestión directiva y los ambientes de aprendizaje acogedores.

H02: No existe relación entre la gestión directiva y los ambientes de aprendizaje acogedores.

Cuadro 13. Correlación entre gestión directiva y ambientes de aprendizaje acogedores.

\begin{tabular}{|c|c|c|}
\hline & Spearman & $\begin{array}{l}\mathrm{D}_{2}: \text { Ambientes de aprendizaje aco- } \\
\text { gedores }\end{array}$ \\
\hline \multirow{3}{*}{$\mathrm{V}_{1}$ : Gestión Directiva } & $\mathrm{r}$ &, $380^{* *}$ \\
\hline & Sig. &, 004 \\
\hline & $\mathrm{n}^{\circ}$ & 46 \\
\hline
\end{tabular}

El análisis de correlación indica que la gestión directiva se relaciona de manera significativa (Sig. <0.05) Con los ambientes de aprendizaje acogedores; este resultado da evidencias suficientes para validar la hipótesis de investigación. En consecuencia, se acepta la hipótesis de investigación. Este resultado permite establecer que en la medida que mejora la gestión directiva escolar mejorará los ambientes de aprendizaje acogedores.
Gestión directiva y ambientes saludables de aprendizaje

Hi3: Existe relación entre la gestión directiva y ambientes saludables de aprendizaje.

H03: No existe relación entre la gestión directiva y ambientes saludables de aprendizaje.

Cuadro 14. Correlación entre gestión directiva y ambientes saludables de aprendizaje.

\begin{tabular}{|c|c|c|}
\hline & Spearman & $\begin{array}{c}\mathrm{D}_{3}: \text { Ambientes saludables de apren- } \\
\text { dizaje }\end{array}$ \\
\hline \multirow{3}{*}{$\mathrm{V}_{1}$ : Gestión Directiva } & $\mathrm{r}$ & ,153 \\
\hline & Sig. & ,309 \\
\hline & $\mathrm{n}^{\circ}$ & 46 \\
\hline
\end{tabular}

El análisis de correlación indica que la gestión directiva no se relaciona de manera significativa (Sig. >0.05) Con los ambientes saludables de aprendizaje; este resultado no da evidencias suficientes para validar la hipótesis de investigación. En consecuencia, se acepta la hipótesis nula. 


\section{DISCUSIÓN}

Desde el enfoque de la Eficacia Escolar, el Clima deaprendizaje según Espinozay Rodríguez (2017), se desarrolla con base en la interacción social, normativa institucional, -instalaciones, recursos y herramienta-, y práctica docente. Para que todos estos elementos interactúen en perfecto funcionamiento se necesita de la intervención de la gestión directiva, entendida como la capacidad del equipo directivo en tomar decisiones colegiadas para gestionar el liderazgo pedagógico, formular el Proyecto Educativo Institucional y plantear el Plan Operativo Anual. En otras palabras, para responder con resultados educativos exitoso requiere de un trabajo fuerte de equipo, donde todos hablen el mismo idioma y donde lo más importante, la educación peruana, se transforme día a día y brinde a sus estudiantes mejores condiciones de vida personal y académica.

La gestión directiva resultó poco eficiente para el 47,8\% de la población según Córdova, Díaz, Vanegas y Báez (2015), se debe apuntalar la gestión directiva para asegurar que los procesos relacionados con el clima de aprendizaje también mejoren.

El clima de aprendizaje fue clasificado con un nivel alto. Ello se debe al nivel alto en las relaciones interpersonales, ambientes de aprendizaje acogedores y ambientes saludables de aprendizaje.

La relación entre la gestión directiva y las relaciones interpersonales obtuvo una correlación significativa encontrada permite establecer que efectivamente ambos aspectos se relacionan, lo que también permite aceptar la hipótesis de investigación de que la gestión directiva está ligada a las relaciones interpersonales. Las relaciones interpersonales son buenas cuando los estudiantes practican el afecto, la justicia, la confianza, el respeto y la colaboración. Molina y Pérez (2006), señala que las relaciones humanas se refieren al trato o la comunicación que se establece entre dos o más personas; son muy importantes en las instituciones escolares, cuando una persona desarrolla las relaciones interpersonales, éstas mejoran sus actitudes en cooperación, receptividad, autonomía, participación y satisfacción.

Se establece la relación entre la gestión directiva y el clima de aprendizaje en la Institución Educativa Federico Helguero Seminario de los Algarrobos, Piura, el estudio encontró efectivamente que ambos aspectos, se encuentran relacionados.

\section{CONCLUSIONES}

Todas las dimensiones de la variable gestión directiva se cumplen con solvencia en relación a la gestión de recursos pedagógicos, atención a las necesidades estudiantiles, gestión de las estrategias didácticas, gestión de la organización escolar, gestión del trabajo administrativo, gestión entre la organización escolar y la comunidad, y el liderazgo escolar.

El clima de aprendizaje se ve favorecido por el desarrollo de las relaciones interpersonales, los ambientes de aprendizaje de acogida y los ambientes saludables de aprendizaje, es decir, los actores educativos reconocen la importancia del clima de aprendizaje como parte de la política institucional escolar.

Existe relación significativa entre la gestión directiva y las relaciones interpersonales que es parte del clima de aprendizaje. Además, se aprecia una correlación positiva que indica que en la medida que mejora la gestión directiva también mejora las relaciones interpersonales, lo a su vez se certifica con $81,0 \%$ de calificación entre el nivel eficiente de la gestión directiva y el nivel alto de las relaciones interpersonales.

Existe relación significativa entre la gestión directiva y los ambientes de aprendizaje acogedores. La correlación positiva en ambos aspectos determina que en la medida que mejora la gestión directiva también mejora los ambientes de aprendizaje acogedores.

No existe relación significativa entre la gestión directiva y los ambientes saludables para 
el aprendizaje. Es importante que los directivos fortalezcan el diálogo frente a los conflictos actuando con criterios éticos, respetando los códigos culturales, fomentando los medios pacíficos.

Existe relación significativa entre la gestión directiva y el clima de aprendizaje.

\section{REFERENCIAS}

Adelman, H. y Taylor, L. (2004). Classroom climate. En S.W. Lee, P.A. Lowe y E. Robinson (Eds.), Encyclopedia of School Psychology. Thousand Oaks, CA: Sage

Blanco, R. (2014). Aulas inclusivas. Organización de Estados Iberoamericanos Disponible:https://educacion.gob.ec/wpcontent/uploads/downloads/2014/07/AulasInclusivas.pdf

Córdova,C., Díaz,S., Vanegas, A., y Báez,J.(2015). Las competencias del directivo docente en la gerencia educativa. Obtenido de Universidad de la Sabana, tesis de especialización en gerencia educativa, Cundinamarca (Colombia). Disponible:https:// intellectum.unisabana.edu.co/bitstream/ handle/10818/19903/Carmen\%20Tulia\%20 Cordoba $\% 20$ Acosta $\% 20 \% 28$ tesis $\% 29$. pdf? sequence $=1$ \&isAllowed $=y$

Duarte, J. (2003) ambientes de aprendizaje una aproximación conceptual. Revista Iberoamericana de Educación

Espinoza, L., y Rodríguez, R. (2017). La generación de ambientes de aprendizaje: un análisis dela percepción juvenil. Rev.Iberoam. Investig. Desarro. Educ, 7(14) Guadalajara ene. /jun. 2017 Disponible: http://www. scielo.org. $\mathrm{mx} / \mathrm{scielo}$.php? script $=$ sci_ arttext\&pid=S200774672017000100110
García, A., y Ferreira, G. (2005). La convivencia escolar en las aulas. International Journal of Developmental and Educational Psychology, 2 (1), 163-183. Disponible: http://www. redalyc.org/pdf/3498/349832309012.pdf

Herrera, K., y Reinaldo R. (2014). El clima escolar como elemento fundamental de la convivencia en la escuela. Revista Escenarios, 7-18

La Escuela Agroecológica de Pirqué de Chile (sf). Manual de gestión directiva en un modelo holístico. Disponible: http:// fundacionorigen.cl/esp/wp-content/ uploads/2011/11/Manual-GestionDirectiva.pdf

Ministerio de Educación del Perú. (2015). Marco de buen desempeño directivo. Lima: MINEDU

Molina, N., y Pérez, I. (2006). El clima de relaciones interpersonales en el aula: Un caso de estudio. Revista, 27(2) diciembre de 2006 1-19. Disponible: file://C:/Users/Juan $\% 20$ Jos\%C3\%A9/Downloads/3770-9005-1-PB. pdf

Ruíz, W., y Gallo, L. (2011). La gestión directiva una herramienta para generar procesos de calidad educativa en el colegio Alejandro Obregón.Disponible:file://C:/ Users/Juan\%20Jos\%C3\%A9/Downloads/ ARTICULO+CIENTIFICO.+POSO.\%20(1)

UNESCO (2013). Análisis del Clima Escolar: Poderoso factor que explica el aprendizaje en América Latina y El Caribe. Publicación, Santiago: Oficina Regional de Educación para América Latina y el Caribe 